\title{
An "In-House" ELISA for SARS-CoV-2 RBD uncovers elevated immune response at higher altitudes
}

Tomas Grau Rodrigo ${ }^{1 \dagger}$, Ploper Diego ${ }^{1 \dagger}$, Ávila César ${ }^{1}$, Vera Pingitore Esteban $^{1}$, Maldonado Carolina ${ }^{2}$, Chaves Silvina ${ }^{1}$, Socias Sergio Benjamín ${ }^{1}$, Stagnetto Agustín ${ }^{1}$, Navarro Silvia ${ }^{1}$, Chahla Rossana ${ }^{3}$, Aguilar Mónica ${ }^{3}$, Llapur Conrado ${ }^{4}$, Aznar Patricia ${ }^{3}$, Alcorta Malena ${ }^{3}$, Costas Dardo ${ }^{3}$, Flores Isolina $^{3}$, Heinze Dar $^{7}$, Apfelbaum Gabriela ${ }^{5}$, Mostoslavsky Raúl ${ }^{6}$, Mostoslavsky Gustavo ${ }^{7}$, Cazorla Silvia ${ }^{2 *}$, Perdigón Gabriela ${ }^{2 *}$, Chehín Rosana $^{1 *}$.

1 Instituto de Medicina Molecular y Celular Aplicada - IMMCA (CONICET-UNT-SiProSa). Tucumán, Argentina.

2 Centro de Referencia para Lactobacilos - CERELA (CONICET). Tucumán, Argentina.

3 Hospital Néstor Kirchner, Laboratorio de Salud Pública, Sistema Provincial de Salud - LSP (SiProSa). Tucumán, Argentina.

4 Dirección de Investigación en Salud. Ministerio de Salud Pública, Tucumán, Argentina

5 Facultad de Medicina - Universidad Nacional de Tucumán. Tucumán, Argentina

6 The Massachusetts General Hospital Cancer Center, Harvard Medical School, Boston, MA, USA.

7 Section of Gastroenterology, Department of Medicine, Center for Regenerative Medicine (CReM), Boston University School of Medicine, Boston, MA, USA.

Correspondence to:

* Rosana Chehin - *Gabriela Perdigón - *Silvia Cazorla

rosanachehin@gmail.com

perdigon@cerela.org.ar

silviainescazorla@gmail.com

$\dagger$ Both contribute equally

Abbreviations:

RBD, Receptor binding domain; ROC, receiver operating characteristic curve; ELISA, Enzyme linked immunosorbent assay; mamsl, meters above median sea level 


\begin{abstract}
The severe acute respiratory syndrome coronavirus-2 (SARS-CoV-2) first reported in Wuhan has caused a global pandemic with dramatic health and socioeconomic consequences. The Coronavirus Disease 2019 (COVID-19) associated represents a challenge for health systems that had to quickly respond developing new diagnostic and therapeutic strategies. In the present work, we developed an "In House" ELISA with high sensitivity (92.2\%), specificity (100\%) and precision (93.9\%), with an area under the ROC curve (AUC) of 0.991, rendering the assay as an excellent serological test to correctly discriminate between SARS-COv-2 infected and non-infected individuals and study population seroprevalence. Among 758 patients evaluated for SARS-CoV-2 diagnosis in the province of Tucumán, Argentina, we found a Pearson correlation coefficient of 0.5048 between antibodies elicited against the RBD and the nucleocapsid (N) antigen. Additionally, 33.6\% of individuals diagnosed with COVID-19 displayed mild levels of RBD-IgG antibodies, while $19 \%$ of the patients showed high antibody titers. Interestingly, patients with SARS-COV-2 infection over 60 years old elicited significantly higher levels of IgG antibodies against RBD compared to younger ones, while no difference was found between women and men. Surprisingly, individuals from a high altitude village displayed statistically significant higher and longer lasting anti-RBD antibodies compared to those from a city at a lower altitude, suggesting that a hypobaric hypoxia-adapted mechanism may act as a protective factor for COVID-19. To our knowledge, this is the first report correlating altitude with increased humoral immune response against SARS-Cov-2 infection.
\end{abstract}




\section{Introduction}

The severe acute respiratory syndrome coronavirus 2 (SARS-CoV-2) virus that emerged in December 2019, with its associated Coronavirus Disease (COVID-19), has caused more than 2 million deaths in 2020 alone ${ }^{1}$. It is projected that without efficient action, COVID19 could infect around $90 \%$ of the world's population and kill over 40 million people. While vaccination efforts continue, the near future holds no end in sight for this pandemic ${ }^{2}$. Diagnostic efficiency continues to be one of the main bottlenecks in the fight against COVID-19. While nucleic acid detection of the SARS-CoV-2 RNA genome is the gold standard test to diagnose infection, measurements of antibody responses give essential knowledge regarding protective immunity due to infection or vaccination. Serological surveillance of anti-SARS-CoV-2 antibodies in the population also provides a crucial tool for designing public health guidelines ${ }^{3}$. This is only possible if serological tests are sufficiently trustworthy, which basically implies the correct election of the technique, the target antigen, and the antibodies to be studied.

The SARS-CoV-2 pandemic has disrupted the worldwide supply chain for many diagnostic equipment and their components, challenging government agencies and private companies in their efforts to acquire reagents, testing kits, personal protective equipment, laboratory devices, vaccines, and any COVID-19-related technology. While this situation has provided fertile ground for the development of new private or state-owned companies that have flourished attempting to resolve this urgent demand, supply is still proving insufficient. This is also the case for kits that measure antibodies against SARS-CoV-2. Therefore, in order to resolve this shortage and support state-led diagnostic efforts, many research laboratories have repurposed their expertise, skilled labor and equipment by developing and assembling in-house testing kits, among other efforts ${ }^{4-7}$.

SARS-CoV-2 serological tests employ enzyme-linked immunosorbent assay (ELISA) ${ }^{8,9}$, immunofluorescence ${ }^{10}$ and lateral flow techniques to detect antibodies directed against the nucleocapsid (N) and/or Spike (S) proteins of the viral proteome ${ }^{11}$. The highly glycosylated $\mathrm{S}$ protein, which plays a key role in the receptor recognition and cell membrane fusion process, is composed of two subunits, $\mathrm{S} 1$ and $\mathrm{S} 2$. The $\mathrm{S} 1$ subunit contains a receptor- 
binding domain (RBD) that recognizes and binds to the host receptor angiotensinconverting enzyme $2(\mathrm{ACE} 2)^{12,13}$. The $\mathrm{N}$ protein plays a vital role in transcription and replication and, due to its abundance, has been suggested to be the ideal antigen for detecting early infections ${ }^{3}$. It has been shown that IgG antibodies targeting $\mathrm{S}$ are more specific, while those directed against $\mathrm{N}$ may be more abundant in the earliest phase of infection ${ }^{14,15}$. The sensitivity and specificity of most serological tests have been validated with samples extracted from patients enduring the acute phase of infection ${ }^{14,15}$. Results from these studies indicate that tests using $\mathrm{N}$ and $\mathrm{S}$ were considered equally sensitive ${ }^{14,15}$. Based on these observations, it has been assumed that determination of antibody responses against either protein would be equally suitable in post-infection phase population-based seroprevalence studies ${ }^{16}$. However anti-S and anti-RBD antibodies correlate better with virus neutralization ${ }^{17-20}$. This has been determined through virus neutralization tests (VNT) (the gold standard for measuring neutralization). Since this assay is labor-intensive and requires a highly trained staff working in BSL3 conditions, it is not appropriate for highthroughput detections. In contrast, ELISA with recombinant S and/or RBD as substrate, show a strong correlation with neutralization assay results ${ }^{20,21}$. High-titer plasma therapy is one of the few clinically proven treatments that prevent COVID-19 in adults. Thus, determining the levels of neutralizing antibodies is fundamental when screening samples for potential plasma donors ${ }^{22}$.

In addition, since most of the vaccines approved to date carry the genetic information of $S$ or its RBD domain, only tests that determine anti-S or anti-RBD antibodies can evaluate individual and population seroconversions triggered by vaccination ${ }^{23,24}$. The use of RBD not only streamlined the diagnostic kit production process but also provides a more specific target for neutralizing antibodies ${ }^{25,26}$. Therefore, determining the titers of anti-S or antiRBD antibodies, instead of anti-N, provides more valuable information.

Taking into context the worldwide demand for antibody testing kits and the importance of measuring the seroconversion in the population, the aim of the present study was to develop an "In House" ELISA to detect anti-RBD antibodies in the SARS-CoV-2 convalescent population from Tucumán-Argentina, in order to contribute to local public health policies. The particular geographical location of Tucumán in the Andean foothills allowed us to compare the anti-RBD titers developed by individuals in low altitude locations in contrast 
to a high-altitude hypobaric hypoxia-adapted population. We herein report that inhabitants of Tafi del Valle, a village situated at 2014 meters above median sea level (mamsl), showed statistically significant higher anti-RBD titers than the population of San Miguel de Tucumán, located at 431 mamsl. To our knowledge, this is the first study showing an increase in the humoral immune response against SARS-CoV-2 in a high-altitude population, providing a novel perspective on the hypothesis of increased resistance to COVID-19 in populations adapted to hypobaric hypoxia ${ }^{27-29}$. 


\section{Materials and Methods}

\section{Recombinant SARS-CoV-2 S RBD-His expression:}

A plasmid encoding a secreted his-tagged SARS-CoV-2 S RBD was obtained as a generous gift from Jared Feldman and Aaron G. Schmidt (Harvard University). The RBD sequence was PCR amplified and subcloned into a pHAGE2-EF1a-IRES-ZsGreen-W lentiviral vector $^{30}$. Lentiviral particles were produced by cotransfecting the lentiviral backbone together with appropriate packaging vectors into HEK293 cells at 70\% confluency in a 100 mm petri dish. HEK293 were grown in high glucose medium supplemented with $10 \%$ fetal bovine serum and antibiotic/antimycotic and PEI $87 \mathrm{kDa}$ was used as the transfection reagent. Culture media containing lentiviral particles was harvested after 24/48 $\mathrm{h}$ and used to transduce a fresh HEK293 culture at 70\% confluency in one well of a 6 well plate. Media was washed after 24 hs. Transduction efficiency was assessed by imaging the fluorescence of ZSGreen, expressed from the pHAGE2 lentiviral vector.

\section{Recombinant SARS-CoV-2 S RBD-His purification:}

Purification of each RBD-His contained in the conditioned media was carried out by affinity chromatography using a $5 \mathrm{ml}$ HisTrap HP column (GE Healthcare, UK) coupled to an Akta Pure 201 (GE) chromatograph (FPLC). Briefly, the supernatant was harvested from the cell culture, supplemented with $20 \mathrm{mM}$ imidazole and filtered through a $0.22 \mathrm{um}$ pore size PVDF filter. The affinity column was equilibrated with 10 column volumes of buffer A (20 mM NaH $2 \mathrm{PO}_{4}, \mathrm{NaCl} 500$ mM, pH 7.4, 20 mM imidazole), loaded with 100 $\mathrm{ml}$ sample using a sample pump, and washed with 10 column volumes of buffer $\mathrm{A}$ at a flow rate of $4 \mathrm{ml} / \mathrm{min}$. Bound proteins were eluted with a step gradient with $5 \mathrm{CV}$ of either $20 \%$, $50 \%$ and $100 \%$ of buffer $\mathrm{B}\left(20 \mathrm{mM} \mathrm{NaH}_{2} \mathrm{PO}_{4}, \mathrm{NaCl} 500 \mathrm{mM}, \mathrm{pH} 7.4,500 \mathrm{mM}\right.$ imidazole). Fractions of $5 \mathrm{ml}$ were collected and further analyzed using SDS-PAGE. Fractions with molecular weight compatible with the His-tagged RBD were pooled and dialyzed against $200 \mathrm{mM} \mathrm{NaH}{ }_{2} \mathrm{PO}_{4}, \mathrm{pH}$ 6.5. The production yield for RBD was around $0.5 \mathrm{mg} / 100 \mathrm{ml}$ of cell culture supernatant, with a purity level higher than $95 \%$. 


\section{ELISA anti-RBD antibody binding assay:}

An indirect ELISA test for the determination of IgG anti-RBD antibody was design. Briefly, flat polystyrene bottom plates (High Binding, Half-Area, Greiner 675061) were sensitized with $0.1 \mu \mathrm{g}$ per well of the RBD antigen for $18 \mathrm{~h}$ at $4^{\circ} \mathrm{C}$. Blocking was done with $10 \%$ Fetal Bovine Serum (FBS) in PBS $10 \mathrm{mM} \mathrm{pH} 7.4$ during $1 \mathrm{~h}$ at $37^{\circ} \mathrm{C}$. Plates were then washed three times with $0.1 \%$ Tween in PBS. Sera were assayed at a serial dilution of $1 / 100$ and incubated for $1 \mathrm{~h}$ at $37^{\circ} \mathrm{C}$. Peroxidase-conjugated immunoglobulins to human IgG (whole molecule) -Peroxidase (Sigma A8667) diluted 1/35,000 were used as a secondary antibody. Plates were developed by adding TMB (3,3',5,5' Tetrametilbenzidina; BD OptEIAtm), incubated for $15 \mathrm{~min}$ in the dark and the reaction was stopped using 4N $\mathrm{H}_{2} \mathrm{SO}_{4}$. Optical density was read by an ELISA reader (TECAN Spark) at $450 \mathrm{~nm}$. Cutoff values were calculated using receiver operating characteristic curve (ROC). Titers were calculated as the dilution in which the optical density (OD) obtained was equal to the cutoff.

\section{Populations studied:}

Serum samples (758) taken in the Laboratorio de Salud Pública- Sistema Provincial de Salud de Tucumán (SIPROSA) from patients with diagnosis of SARS-Cov-2 infection by positivity results in the polymerase chain reaction (PCR) or due to close contact to SARSCov-2 infected peoples were analyzed. Serum samples were taken at least 3 weeks after the diagnosis of Covid-19. The procedures were approved by the Bioethics Committee of the SIPROSA N ${ }^{\circ} 29 / 2020$. As control of no SARS-Cov-2 infection 26 serum taken before December of 2019 were analyzed (prepandemic serum). RBD specific IgG levels along time was evaluated in 574 and 14 patients, from a high-altitude village (Tafí del Valle, population 14933, representing $0.093 \%$ of the population), and a lower altitude city (San Miguel de Tucumán, population 830000 , representing $0.069 \%$ of the population), respectively, with SARS-CoV-2 infection confirmed by RT-PCR.

\section{Statistical analyses:}

The cutoff point for optimal sensitivity and specificity, as well as the other statistical parameters, were determined using the receiver operating characteristic (ROC) curve 
medRxiv preprint doi: https://doi.org/10.1101/2021.03.10.21252711; this version posted March 12, 2021. The copyright holder for this preprint (which was not certified by peer review) is the author/funder, who has granted medRxiv a license to display the preprint in perpetuity. All rights reserved. No reuse allowed without permission.

analysis using the XL-STAT statistical software/program (Excel). RBD-IgG titers, and correlation and differences between medias studies were carried out with the Prisma 8.0 Software (GraphPad, San Diego, CA). For non-parametric variables, data were analysed by the Kruskal-Wallis test. Significant differences between groups are shown with the corresponding P values. Significant differences are indicated with asterisks. 


\section{Results}

1) Expression and purification of the recombinant RBD-SARS-CoV-2. In order to evaluate local population anti-SARS-CoV-2 IgG antibody levels, an "In House" ELISA assay for SARS-CoV-2 which uses the RBD of S was developed. For this, a transgenic HEK293 cell line was produced that expresses and secretes the His-tagged RBD (HEK293RBD-His-SARS-CoV-2). This stable cell line, which secretes large amounts of the RBDHis into the culture medium, was achieved by transducing HEK293 cells with a pHAGE2 lentivirus (pHAGE-RBD-His), generated by cotransfecting HEK293 cells with the appropriate lentiviral vector and corresponding packaging plasmids (materials and methods) (Fig. 1A-C). The secreted RBD-His domain, efficiently purified by affinity chromatography, migrated as expected on an SDS-PAGE gel according to previous reports (Fig. 1D-E) ${ }^{31}$.

\section{2) Diagnostic performance of an "In House" ELISA using purified SARS-CoV-2}

RBD. A titration curve was performed to determine the most appropriate concentration of the RBD antigen to be used in the ELISA (data not shown). Then, ELISA were performed to determine the titer of specific IgG antibodies against RBD in serum samples from convalescent serum of patients of the local state health system (SIPROSA-Tucumán, Argentina). As positive controls for SARS-CoV-2 infection, we analysed 52 patients that presented positive results both in the PCR test and in the Chemiluminescent microparticle immunoassay (CMIA-Architect, Abbot) performed on the nasopharyngeal swab and on serum samples, respectively. As shown in Fig. 2A, anti-RBD IgG antibody levels were significantly higher in samples from these patients previously diagnosed with SARS-CoV2 than in serum samples extracted from pre-pandemic patients $(\mathrm{p}<0.0001)$ (Fig. 2A).

Next, we analysed the accuracy of our ELISA test to correctly classify samples as positive for SARS-CoV-2 infection. As shown in Fig. 2B, the AUC 0.991 (95\% confidence interval 0.9778 to 1.000 ) was determined for the RBD antigen. Moreover, the ELISA for the determination of IgG anti-RBD presented high sensitivity (92.2\%), specificity (100\%) and also a predictive positive value (1.00) (Fig. 2C). In order to determine if antibodies present in serum elicited by other microorganisms can interfere with the ELISA test, sera extracted 
from patients before December 2019 with human immunodeficiency virus (HIV), Toxoplasma gondii, Trypanosoma cruzi, and human hepatitis A virus (HAV) infections were tested. As shown in Fig. 2D, no false positive results arose from these samples $(* * * *$ $\mathrm{p}<0.0001)$.

\section{3) Performance of an "In House" ELISA compared to a commercially available test.}

The ability to detect antibodies triggered by SARS-CoV-2 infection was compared between the anti-RBD ELISA and a commercially available Chemiluminescent Microparticle ImmunoAssay (CMIA) kit that detects antibodies against the $\mathrm{N}$ protein (Architect, Abbott). Among 595 patients that were classified as SARS-CoV-2-positive by RT-PCR from the local public health system, we observed that the ELISA and the CMIA-based Architect displayed similar diagnostic abilities, 59\% and 58.3\%, respectively (Fig. 3A). Interestingly, similar results were observed in patients referred to as close contacts to SARS-CoV-2 infected patients, as both tests were able to detect antibodies in similar rates within this population (anti-RBD ELISA, 63.8\%; anti-N CMIA, $58.9 \%$ ) (Fig. 3A).

We further analysed the correlation between both tests, and found a Pearson correlation coefficient of 0.5048 between anti-RBD IgG titers and the anti-N IgG index (Fig. 3B). Albeit this weak correlation between the titers of anti-RBD and the CMIA index of anti-N antibodies, a high concordance for the presence or absence of both antibodies was observed (Fig 3C). Therefore, as shown in Fig. 3B, high anti-N IgG antibodies in a given patient does not necessarily reflect stimulation of high humoral immune response against RBD. This fact could be due to differences in antigen expression levels or time elapsed after infection. Overall, these results endorse the "In House" ELISA for the detection of antiRBD IgG as an excellent candidate for the determination of humoral immune responses elicited against SARS-Cov-2 infection.

\section{4) Distribution of anti-RBD specific IgG antibodies measured with an "In House" ELISA among patients diagnosed with SARS-CoV-2.}

In order to study the distribution of anti-RBD IgG titers among true positive samples, we analysed, with the "In House" ELISA, the sera of 366 patients previously diagnosed with SARS-CoV-2 by RT-PCR and who also tested positive for anti-N antibodies by CMIA. 
The distribution of antibody levels showed a preponderance of titers between 300 and 800 (37\%), where $19 \%$ of titers are above 1350 (Fig. 4A). When 758 patients that tested positive for CMIA anti-N were analysed by the "In House" ELISA and segregated into three arbitrary age groups, $>40$ years, $40-60$ years and $<60$ years, we found that IgG anti-RBD antibodies were significantly elevated in the over-60 age group, as previously reported ${ }^{32}$. No significant differences were found in average RBD-specific IgG titers between male and female patients (Fig. 4C), although the percentage of negativity was much higher in males $(48.9 \%)$ than in females $(9.7 \%)$ in the studied population with the anti-RBD ELISA. Overall, the distribution of RBD-specific IgG antibodies in the population of Tucumán was found to be consistent with what has been described in previous seroprevalence reports 33,34 .

\section{5) Increased and long-lasting anti-RBD humoral immune response in a high altitude population.}

In addition to serum samples corresponding to patients from the city of San Miguel de Tucumán (431 mamsl), we also measured anti-RBD IgG titers in patients from Tafí del Valle, a small village located at 2014 mamsl. Surprisingly, we observed significantly higher anti-RBD IgG antibody levels in patients from this high altitude village compared to the lower altitude population $(\mathrm{p}<0.001)$ (Fig. 5A). Interestingly, high altitude patients sustained specific antibody titers for extended periods of time, showing similar IgG specific antibodies levels at day 90 and 30 post COVID-19. By contrast IgG anti-RBD IgG antibodies significantly decrease at day 90 post-diagnosis of viral infection with respect to the levels observed at day 30 in individuals from the lower altitude population studied. The fact that this ratio of antibodies between day 90 and 30 in these two populations show significant differences $(\mathrm{p}<0.005)$ (Fig. 5B), suggests that a hypobaric hypoxia-adapted mechanism might play a key role in the SARS-CoV-2 humoral immune response. 


\section{Discussion}

The new coronavirus (SARS-CoV-2) infection has reached every continent, with new variants spreading quickly. As second and third waves of infections arise, and vaccination efforts attempt to ramp up, there is no consensus as to how long this pandemic could last. Among patients infected with SARS-CoV-2, the progression of disease is highly variable. Eighty percent of patients that become infected develop mild or no symptoms; whereas the remaining $20 \%$ develop moderate to severe disease ${ }^{35,36}$. SARS-CoV-2 pathogenicity, results from an acute excessive virus replication followed by an uncontrolled inflammation and an exacerbated immunity. As the virus replicates, the adaptive immunity is stimulated to generate cellular and humoral responses in order to control the viral infection.

The role of sensitive molecular diagnostic techniques, such as RT-PCR and rapid antigen tests, are essential for the diagnosis of SARS-CoV-2 infection. Nevertheless, immunoserological tests have evolved as an indispensable tool, for example, in screening of potential plasma donors with high titers of anti-SARS-CoV-2 neutralizing antibodies, since the proven success of convalescent plasma therapy for COVID-19 ${ }^{4}$. Many approaches have demonstrated that protection against SARS-CoV-2 is positively correlated with the development of high titers of neutralizing antibodies ${ }^{17-20}$. Due to its role in viral entry to the host cell, the RBD of S emerged as a potential target antigen for the development of preventive and therapeutic strategies against COVID-19 ${ }^{20,37}$. Equally as important is the usefulness of RBD for the diagnosis of SARS-Cov-2 infection, as supported by epidemiological data and molecular diagnosis 33,34,38. However, the unavailability of sensible, robust and cost-effective anti-RBD IgG diagnostic kit in our region motivated us to develop an "In House" ELISA for the detection of anti-RBD humoral responses against SARS-CoV-2.

By creating a stable cell line expressing high levels of RBD-His we were able to ensure a sufficient amount of antigen that enabled us to test thousands of patients from the public health system (SIPROSA-Tucumán, Argentina) (Fig 1). The immobilization of this antigen to ELISA plates allowed for the assembly of a highly sensitive and specific ELISA assay that showed an AUC of 0.991, for the detection of anti-RBD IgG elicited after SARS-CoV2 infection. According to the traditional academic point system, an AUC between 0.90-1.0 
indicates the antigen is an excellent ligand to correctly discriminate between the two groups (infected and non-infected) ${ }^{39}$. The ELISA test developed did not show cross reactivity with pre-pandemic sera from patients infected with other common infections.

Importantly, the ability of the "In House" ELISA to correctly discriminate the occurrence or not of a SARS-CoV-2 infection was compared with other molecular and serological tests. We observed that RBD specific IgG antibodies were elicited in $60 \%$ of patients, previously diagnosed as SARS-CoV-2 positive. It has been reported that following infection, antibodies directed against RBD and $\mathrm{N}$ antigens begin to be detectable at slightly different times and in different amounts ${ }^{40,41}$. Therefore, high levels of antibodies elicited against one antigen do not imply the presence of similar amounts of the other (Fig. 2). Nevertheless, our "In House" ELISA test showed high concordance with the commercial CMIA Architect by Abbott in discriminating presence or absence of IgG antibodies against SARS-CoV-2.

Our "In House" ELISA allowed surveying the immune response against SARS-CoV-2 $\mathrm{RBD}$ in patients of the public health system. Figure $4 \mathrm{~A}$ revealed that $19 \%$ of individuals previously infected with SARS-CoV-2 displayed titers above 1350. According to Salazar et al. ${ }^{21}$, these values correlate to a probability $\geq 80 \%$ of having neutralizing titers 160 . Our data showed that one out of five plasma from COVID-19 recovered individuals were suitable candidates as donor for convalescent plasma therapy. Elevated anti-RBD IgG titers were found in patients above 60 years old compared to under 40 and $40-60$ age groups. We also found that only $9.7 \%$ out of 196 female patients tested negative for anti-RBD IgG, compared to $48.9 \%$ in the case of males. This is in accordance with the fact that female sex is associated with greater SARS-CoV-2 antibody levels in disease early phase ${ }^{42}$. However, the average titers induced by each sex group showed no statistical difference. Among the population studied, we noted that a high proportion of samples extracted from patients that reside in high altitude villages showed high anti-RBD IgG antibodies. Further analysis confirmed that patients from Tafí del Valle, a mountain village of 15000 residents situated at 2014 mamsl, presented increased levels of antibodies against RBD compared to patients from San Miguel de Tucumán, located at a much lower elevation (431 mamsl). To our knowledge, this is the first report that correlates high titers of anti-RBD antibodies with altitude. This suggests that a hypobaric hypoxia-adapted mechanism may play, at least in 
part, an important role in triggering a long-lasting humoral immune response of SARS$\mathrm{CoV}-2$, and might help explain previous publications reporting altitude as a protective factor for COVID-19 29 27,28. These results provide an important quantitative target for therapeutic and prophylactic treatments.

\section{Figure Legends}

Figure 1. Expression and purification of the recombinant RBD-SARS-CoV-2. (A-C) Highly efficient transduction of HEK293 cells with pHAGE2 lentiviral particles coding for a secreted form of RBD-His can be observed by expression of the fluorescent reporter ZsGreen, co-expressed from the same lentivirus. D) Elution profile of the supernatant from the stable transgenic cell line HEK293-RBD-His-SARS-CoV-2 produced. The purified protein appeared in the second absorbance peak (blue) as indicated (arrow). E) SDSPAGE of RBD-His purified by an HPLC system using HisTrap columns. Lane 1: purified RBD showing the expected molecular weight; lane 2: molecular weight marker.

Figure 2. Diagnostic performance of an "In House" ELISA using purified SARS-CoV-2 RBD. A) ELISA test for IgG antibodies against RBD. Serum samples were classified as positive for SARS-CoV-2 infection by being positive in the PCR and the CMIA test. Negative samples for SARS-CoV-2 infection were serum taken before December 2019. The results were expressed as the OD450 nm, and the cutoff (CO) was calculated using the ROC curve. B) Diagnostic efficacy of the RBD antigens in SARS-CoV-2 infection using ROC curves. C) Statistic parameters of the ELISA test developed against the RBD antigen. D) IgG antibodies against RBD in sera from patients with infections by: HIV: human immunodeficiency virus; T. gondii: Toxoplasma gondii; T. cruzi: Trypanosoma cruzi; and HAV: human hepatitis a virus $(* * * * \mathrm{p}<0.0001)$.

Figure 3. Performance of the anti-RBD "In House" ELISA compared to a commercially available test. A) Percentages of the population studied ( $\mathrm{n}=758$ ), either diagnosed as SARS-CoV-2 positive by RT-PCR or close contacts of infected patients, that have detectable SARS-CoV-2 anti-RBD or anti-N antibodies as measured by the "In House" ELISA or CMIA, respectively. B) Scatterplot depicting the relationship between the titers from the anti-RBD ELISA and the anti-N CMIA index for 758 patients. C) Concordance or discordance in results from the anti-RBD ELISA and the anti-N CMIA tests in the screening of IgG antibodies elicited after SARS-CoV-2 infection.

Figure 4. IgG anti-RBD antibody titers among patients measured by an "In House" ELISA. A) Distribution of anti-RBD titers among 366 true positive samples. Dot line delimits the population that correlates to a probability $\geq 80 \%$ of having neutralizing titers $\geq 160^{21}$. B) Distribution of anti-RBD titers of 758 patients segregated into three age groups, patients under $40(<40), 40-60$, and above $60(>60)$ years old. 
C) Distribution of anti-RBD titers according to sex. D) Chart detailing the number (n) and percentage (\%) of male or female patients who tested positive or negative for the presence of anti-RBD antibodies in an "In House" ELISA.

Figure 5. High and long lasting anti-RBD humoral response in a high altitude population. Populations at 431 mamsl (low altitude) and 2014 mamsl (high altitude) were assayed for the presence of IgG antibodies elicited against the RBD at 30 and 90 days post-diagnosis by RT-PCR for SARS-CoV-2. A) Specific IgG titers elicited at day 30 post-diagnosis in each population. Graph represent the mean \pm S.E.M. ${ }^{* *}<$ p 0.001 .

B) Evolution of humoral immune response against SARS-CoV-2 after 90 days post-diagnosis. Results represent the ratio between RBD-specific IgG titers at day 90 and day 30 post-diagnosis.

\section{Acknowledges}

This work was supported by grants from SkyBIO LLC, the School of Medicine of the UNT and Tucumán Goverment. We also acknowledge the partial support from Argentinean Research Council-CONICET (PIP 722 and 806), Argentinean Research Agency (PICTMINCYT3379, and PICT2018-02989), Tucuman National University Grant (PIUNT-UNT D644/1 and D624). Diego Ploper was supported by Fundación Florencio Fiorini award We are particularly grateful to Dra. María Gabriela Simesen de Bielke, Graciela de Gorostiza from the Central Blood Bank of Tucumán (Banco Central de Sangre de Tucumán) for providing prepandemic serum samples. We are also thankful to Dr. Mateo Martinez and Dra. Roxana Toledo from the School of Medicine (UNT), and to Ing. Luis Rocha, Ing. Marina Gandur and Dr. Christian Jaroszewski from Medical Technology Department (SiProSa), Farm. Betina Heredia from Sterilization Department from Hospital del Niño Jesús (SiProSa), and CITED (Cámara de Instituciones Educ. Terap. Disc.) for their valuable contribution to the development of the present work.

\section{References}

1. Koh HK, Geller AC, VanderWeele TJ. Deaths From COVID-19. JAMA. 2021;325(2):133-134

2. Zhou P, Yang XL, Wang XG, et al. A pneumonia outbreak associated with a new coronavirus of probable bat origin. Nature. 2020;579(7798):270-273.PMC7095418

3. Burbelo PD, Riedo FX, Morishima C, et al. Sensitivity in Detection of Antibodies to Nucleocapsid and Spike Proteins of Severe Acute Respiratory Syndrome Coronavirus 2 in Patients With Coronavirus Disease 2019. J Infect Dis. 2020;222(2):206213.PMC7313936 
4. Ojeda DS, Gonzalez Lopez Ledesma MM, Pallarés HM, et al. Emergency response for evaluating SARS-CoV-2 immune status, seroprevalence and convalescent plasma in Argentina. PLoS Pathog. 2021;17(1):e1009161.PMC7808630

5. Alandijany TA, El-Kafrawy SA, Tolah AM, et al. Development and Optimization of Inhouse ELISA for Detection of Human IgG Antibody to SARS-CoV-2 Full Length Spike Protein. Pathogens. 2020;9(10).PMC7601663

6. Sil BK, Jahan N, Haq MA, et al. Development and performance evaluation of a rapid inhouse ELISA for retrospective serosurveillance of SARS-CoV-2. PLoS One. 2021;16(2):e0246346.PMC7853452

7. MacMullan MA, Ibrayeva A, Trettner K, et al. ELISA detection of SARS-CoV-2 antibodies in saliva. Sci Rep. 2020;10(1):20818.PMC7705674

8. To KK, Tsang OT, Leung WS, et al. Temporal profiles of viral load in posterior oropharyngeal saliva samples and serum antibody responses during infection by SARSCoV-2: an observational cohort study. Lancet Infect Dis. 2020;20(5):565574.PMC7158907

9. Guo L, Ren L, Yang S, et al. Profiling Early Humoral Response to Diagnose Novel Coronavirus Disease (COVID-19). Clin Infect Dis. 2020;71(15):778-785.PMC7184472

10. Wölfel R, Corman VM, Guggemos W, et al. Virological assessment of hospitalized patients with COVID-2019. Nature. 2020;581(7809):465-469

11. Li Z, Yi Y, Luo X, et al. Development and clinical application of a rapid IgM-IgG combined antibody test for SARS-CoV-2 infection diagnosis. J Med Virol. 2020;92(9):1518-1524.PMC7228300

12. Walls AC, Park YJ, Tortorici MA, Wall A, McGuire AT, Veesler D. Structure, Function, and Antigenicity of the SARS-CoV-2 Spike Glycoprotein. Cell. 2020;183(6):1735.PMC7833104

13. Letko M, Marzi A, Munster V. Functional assessment of cell entry and receptor usage for SARS-CoV-2 and other lineage B betacoronaviruses. Nat Microbiol. 2020;5(4):562569.PMC7095430

14. Zhao J, Yuan Q, Wang H, et al. Antibody Responses to SARS-CoV-2 in Patients With Novel Coronavirus Disease 2019. Clin Infect Dis. 2020;71(16):2027-2034.PMC7184337

15. Kohmer N, Westhaus S, Rühl C, Ciesek S, Rabenau HF. Brief clinical evaluation of six high-throughput SARS-CoV-2 IgG antibody assays. J Clin Virol. 2020;129:104480.PMC7263247

16. Jaworski JP. Neutralizing monoclonal antibodies for COVID-19 treatment and prevention. Biomed J. 2020.PMC7685954

17. Garcia-Beltran WF, Lam EC, Astudillo MG, et al. COVID-19-neutralizing antibodies predict disease severity and survival. Cell. 2021;184(2):476-488.e411.PMC7837114

18. Ju B, Zhang Q, Ge J, et al. Human neutralizing antibodies elicited by SARS-CoV-2 infection. Nature. 2020;584(7819):115-119

19. Barnes CO, Jette CA, Abernathy ME, et al. SARS-CoV-2 neutralizing antibody structures inform therapeutic strategies. Nature. 2020;588(7839):682-687

20. Premkumar L, Segovia-Chumbez B, Jadi R, et al. The receptor binding domain of the viral spike protein is an immunodominant and highly specific target of antibodies in SARS-CoV-2 patients. Sci Immunol. 2020;5(48).PMC7292505

21. Salazar E, Kuchipudi SV, Christensen PA, et al. Convalescent plasma anti-SARS-CoV-2 spike protein ectodomain and receptor binding domain IgG correlate with virus neutralization. J Clin Invest. 2020

22. Libster R, Pérez Marc G, Wappner D, et al. Early High-Titer Plasma Therapy to Prevent Severe Covid-19 in Older Adults. N Engl J Med. 2021.PMC7793608

23. Fu Y, Pan Y, Li Z, Li Y. The Utility of Specific Antibodies Against SARS-CoV-2 in Laboratory Diagnosis. Front Microbiol. 2020;11:603058.PMC7838213 
24. Lee CY, Lin RTP, Renia L, Ng LFP. Serological Approaches for COVID-19:

Epidemiologic Perspective on Surveillance and Control. Front Immunol.

2020;11:879.PMC7194125

25. Berry JD, Hay K, Rini JM, et al. Neutralizing epitopes of the SARS-CoV S-protein cluster independent of repertoire, antigen structure or mAb technology. MAbs. 2010;2(1):53-66.PMC2828578

26. Liu L, Wang P, Nair MS, et al. Potent neutralizing antibodies against multiple epitopes on SARS-CoV-2 spike. Nature. 2020;584(7821):450-456

27. Arias-Reyes C, Zubieta-DeUrioste N, Poma-Machicao L, et al. Does the pathogenesis of SARS-CoV-2 virus decrease at high-altitude? Respir Physiol Neurobiol. 2020;277:103443.PMC7175867

28. Huamaní C, Velásquez L, Montes S, Miranda-Solis F. Propagation by COVID-19 at high altitude: Cusco case. Respir Physiol Neurobiol. 2020;279:103448.PMC7207123

29. Pun M, Turner R, Strapazzon G, Brugger H, Swenson ER. Lower Incidence of COVID19 at High Altitude: Facts and Confounders. High Alt Med Biol. 2020;21(3):217-222

30. Mostoslavsky G, Fabian AJ, Rooney S, Alt FW, Mulligan RC. Complete correction of murine Artemis immunodeficiency by lentiviral vector-mediated gene transfer. Proc Natl Acad Sci U S A. 2006;103(44):16406-16411

31. Mehalko J, Drew M, Snead K, et al. Improved production of SARS-CoV-2 spike receptor-binding domain (RBD) for serology assays. Protein Expr Purif. 2021;179:105802.PMC7687410

32. Li K, Huang B, Wu M, et al. Dynamic changes in anti-SARS-CoV-2 antibodies during SARS-CoV-2 infection and recovery from COVID-19. Nat Commun. 2020;11(1):6044.PMC7699636

33. Figueiredo-Campos P, Blankenhaus B, Mota C, et al. Seroprevalence of anti-SARS-CoV2 antibodies in COVID-19 patients and healthy volunteers up to 6 months post disease onset. Eur J Immunol. 2020;50(12):2025-2040.PMC7756220

34. Lai CC, Wang JH, Hsueh PR. Population-based seroprevalence surveys of anti-SARSCoV-2 antibody: An up-to-date review. Int J Infect Dis. 2020;101:314-322.PMC7546669

35. Chen N, Zhou M, Dong X, et al. Epidemiological and clinical characteristics of 99 cases of 2019 novel coronavirus pneumonia in Wuhan, China: a descriptive study. Lancet. 2020;395(10223):507-513.PMC7135076

36. Cao B, Wang Y, Wen D, et al. A Trial of Lopinavir-Ritonavir in Adults Hospitalized with Severe Covid-19. N Engl J Med. 2020

37. Traugott M, Aberle SW, Aberle JH, et al. Performance of Severe Acute Respiratory Syndrome Coronavirus 2 Antibody Assays in Different Stages of Infection: Comparison of Commercial Enzyme-Linked Immunosorbent Assays and Rapid Tests. J Infect Dis. 2020;222(3):362-366.PMC7542676

38. Huang AT, Garcia-Carreras B, Hitchings MDT, et al. A systematic review of antibody mediated immunity to coronaviruses: kinetics, correlates of protection, and association with severity. Nat Commun. 2020;11(1):4704.PMC7499300

39. Simundic AM, Bölenius K, Cadamuro J, et al. [Joint EFLM-COLABIOCLI recommendation for venous blood sampling]. Ann Biol Clin (Paris). 2019;77(2):131-154

40. Van Elslande J, Decru B, Jonckheere S, et al. Antibody response against SARS-CoV-2 spike protein and nucleoprotein evaluated by four automated immunoassays and three ELISAs. Clin Microbiol Infect. 2020;26(11):1557.e1551-1557.e1557.PMC7834107

41. Liu W, Liu L, Kou G, et al. Evaluation of Nucleocapsid and Spike Protein-Based Enzyme-Linked Immunosorbent Assays for Detecting Antibodies against SARS-CoV-2. J Clin Microbiol. 2020;58(6).PMC7269413

42. Zeng F, Dai C, Cai P, Wang J, Xu L, Li J, Hu G, Wang Z, Zheng F, Wang L. A comparison study of SARS-CoV-2 IgG antibody between male and female COVID-19 
medRxiv preprint doi: https://doi.org/10.1101/2021.03.10.21252711; this version posted March 12, 2021. The copyright holder for this preprint (which was not certified by peer review) is the author/funder, who has granted medRxiv a license to display the preprint in perpetuity.

All rights reserved. No reuse allowed without permission.

patients: A possible reason underlying different outcome between sex. J Med Virol. 2020 Oct;92(10):2050-2054. doi: 10.1002/jmv.25989. Epub 2020 May 22. PMID: 32383183; PMCID: PMC7267228. 
Fig. 1
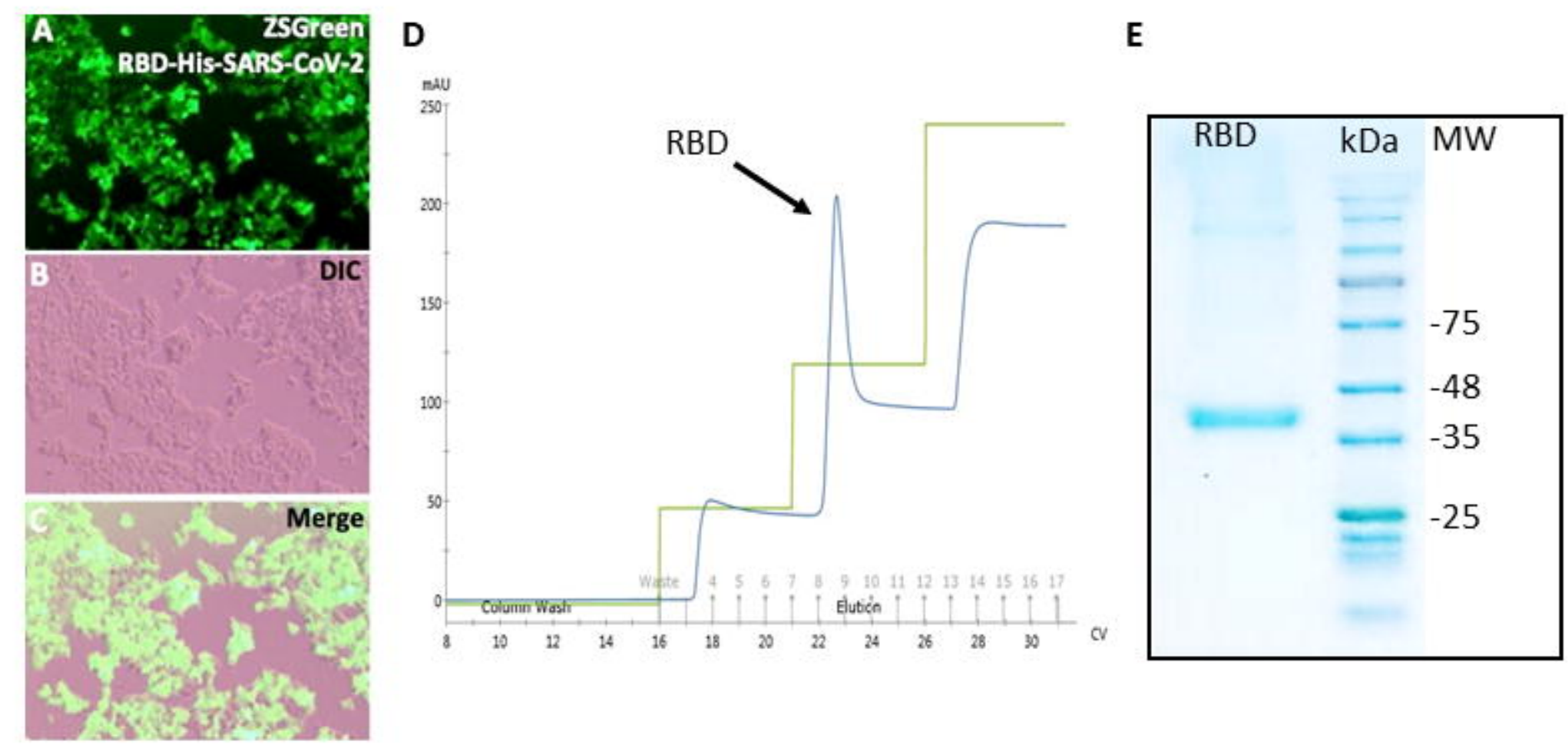
Fig. 2 A

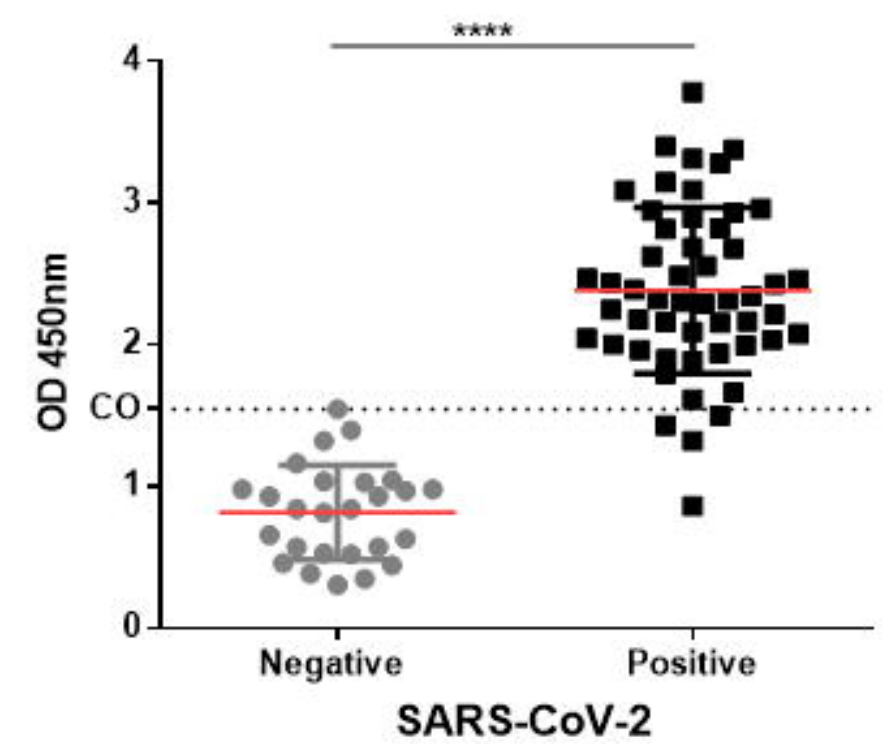

C

ELISA for determination of RBD-specific IgG

\begin{tabular}{|l|c|}
\hline \multicolumn{1}{|c|}{ STATISTIC } & VALUES \\
\hline Sensitivity & 0.923 \\
Specificity & 1.00 \\
True positives & 48 \\
False positives & 0 \\
True negatives & 25 \\
False negatives & 4 \\
Positive predictive value & 1.00 \\
Negative predictive value & 0.862 \\
Precision & 0.948 \\
& \\
\hline
\end{tabular}

B

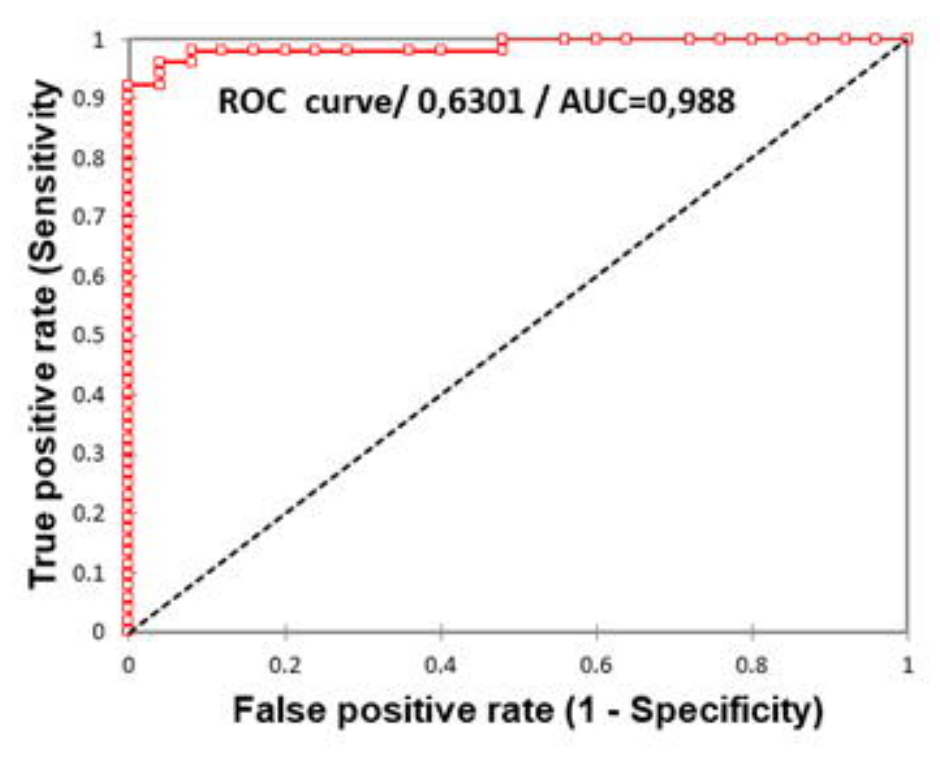

D

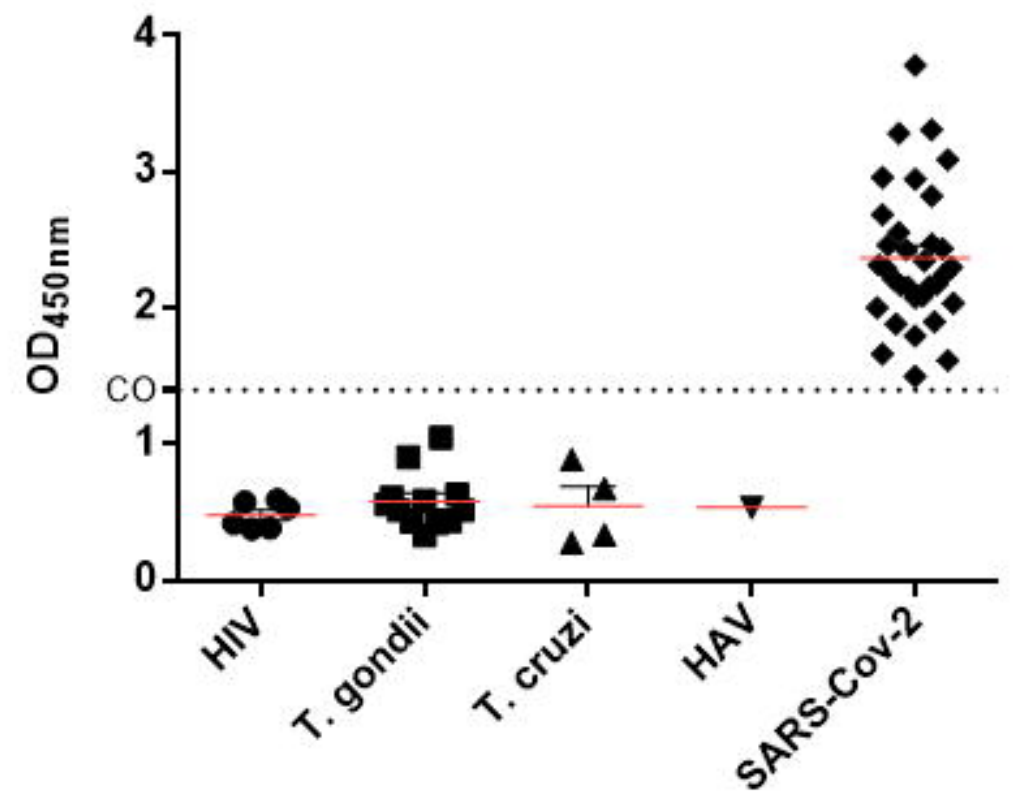


Fig. 3

A

\begin{tabular}{|c|c|c|c|c|}
\hline \multirow{5}{*}{ 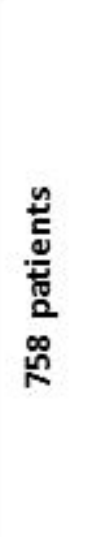 } & & IgG a-RBD (+) & IgGa-N (+) & $\operatorname{IgG}$ a-RBD $(+)$ and IgG a-N (+) \\
\hline & PCR (+) & $59 \%$ & $58.3 \%$ & $47.9 \%$ \\
\hline & $n=595$ & $n=351$ & $n=347$ & $n=285$ \\
\hline & Close contact & $63.8 \%$ & $58.9 \%$ & $49.7 \%$ \\
\hline & $n=163$ & $n=104$ & $n=96$ & $n=81$ \\
\hline
\end{tabular}

B

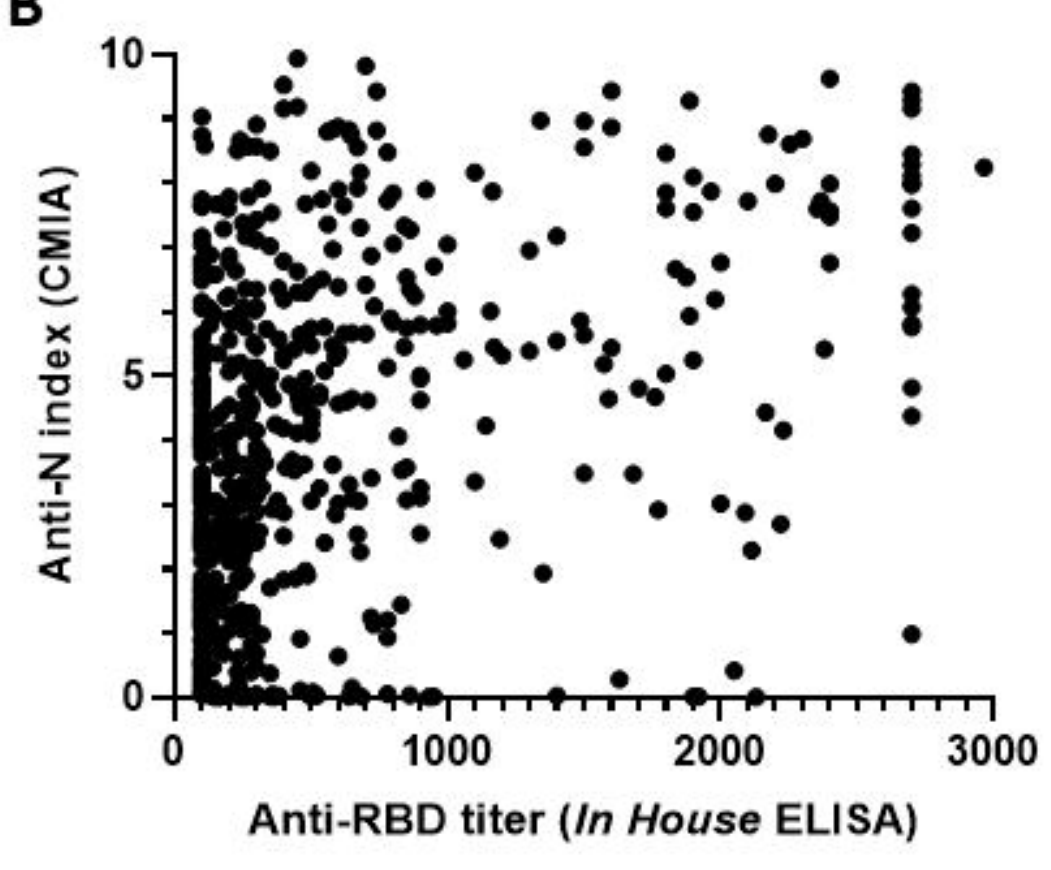

C

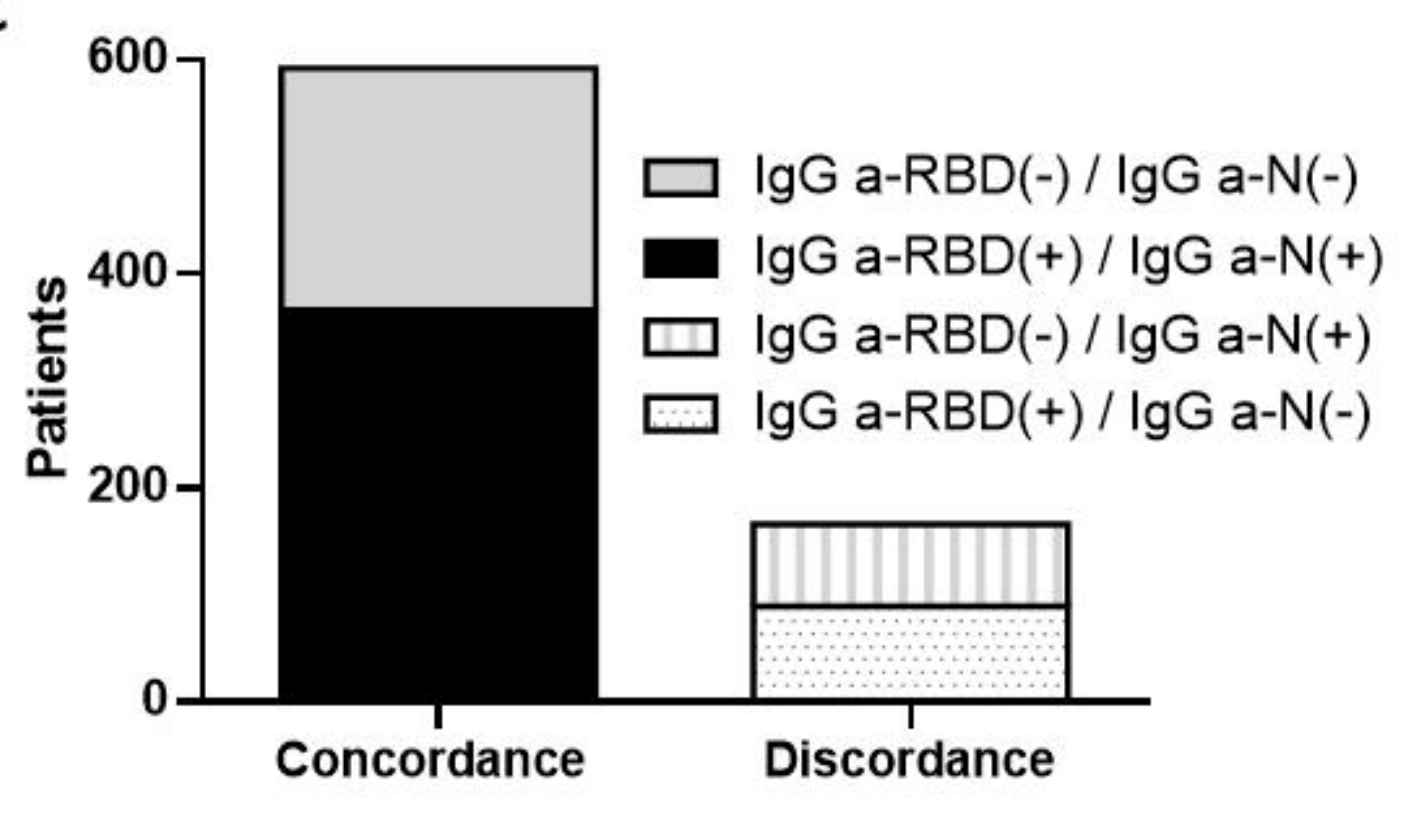


Fig. 4

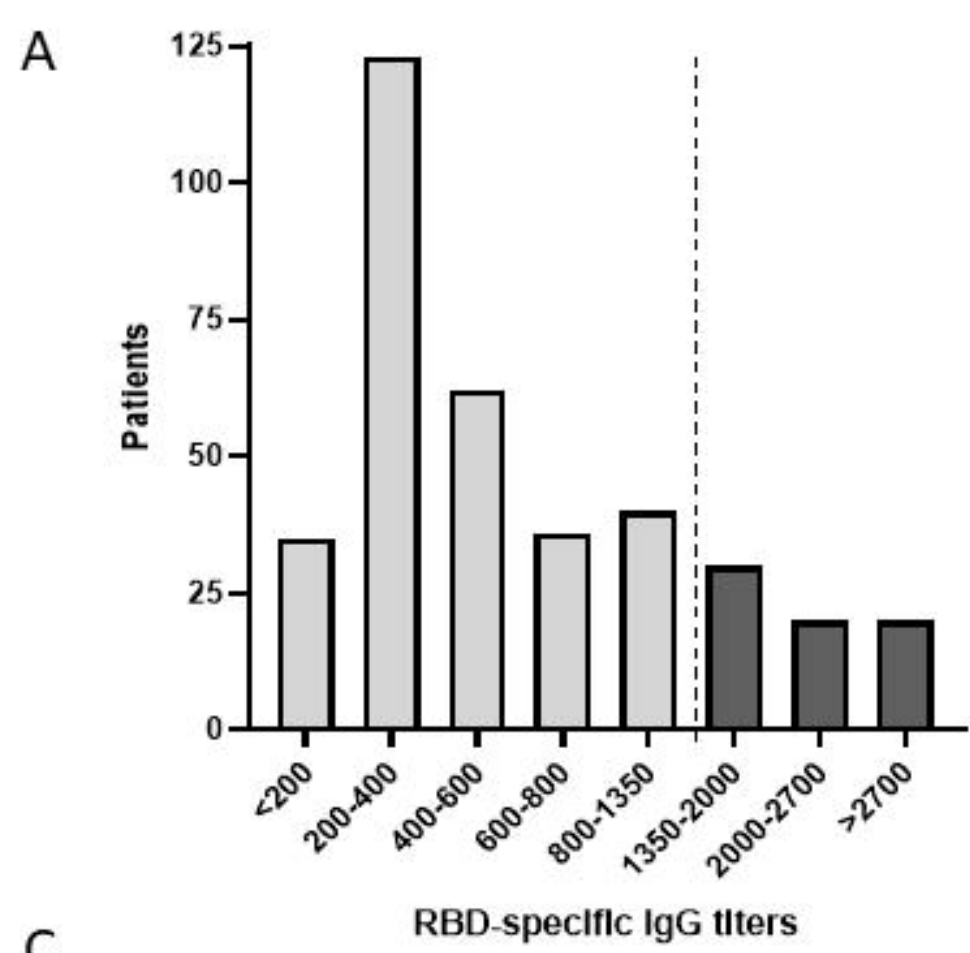

C

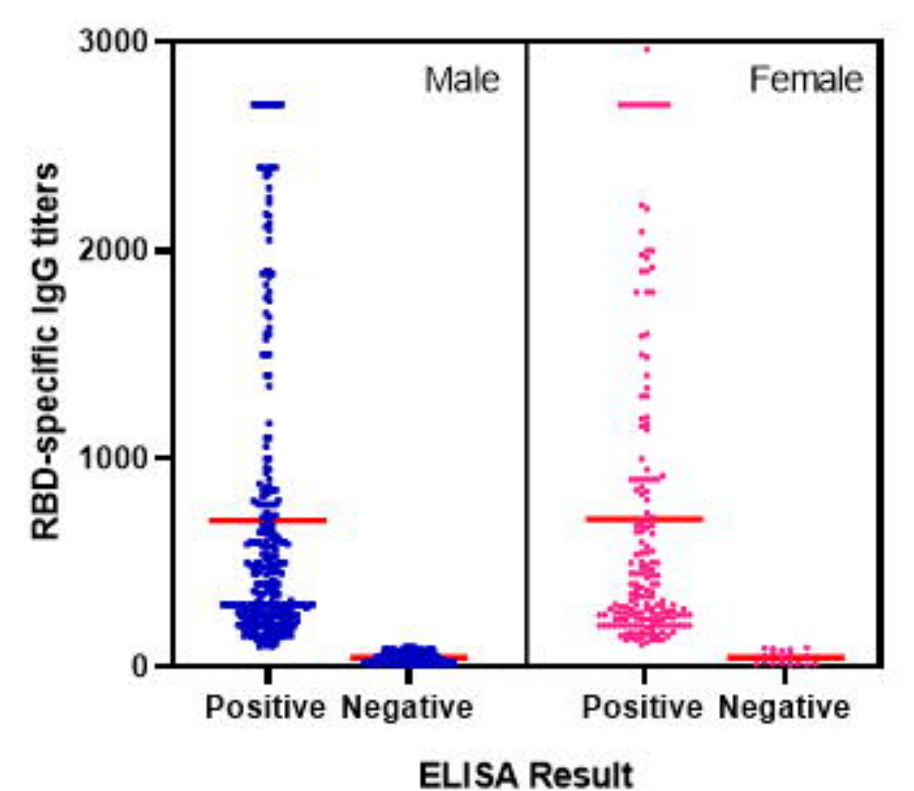

B

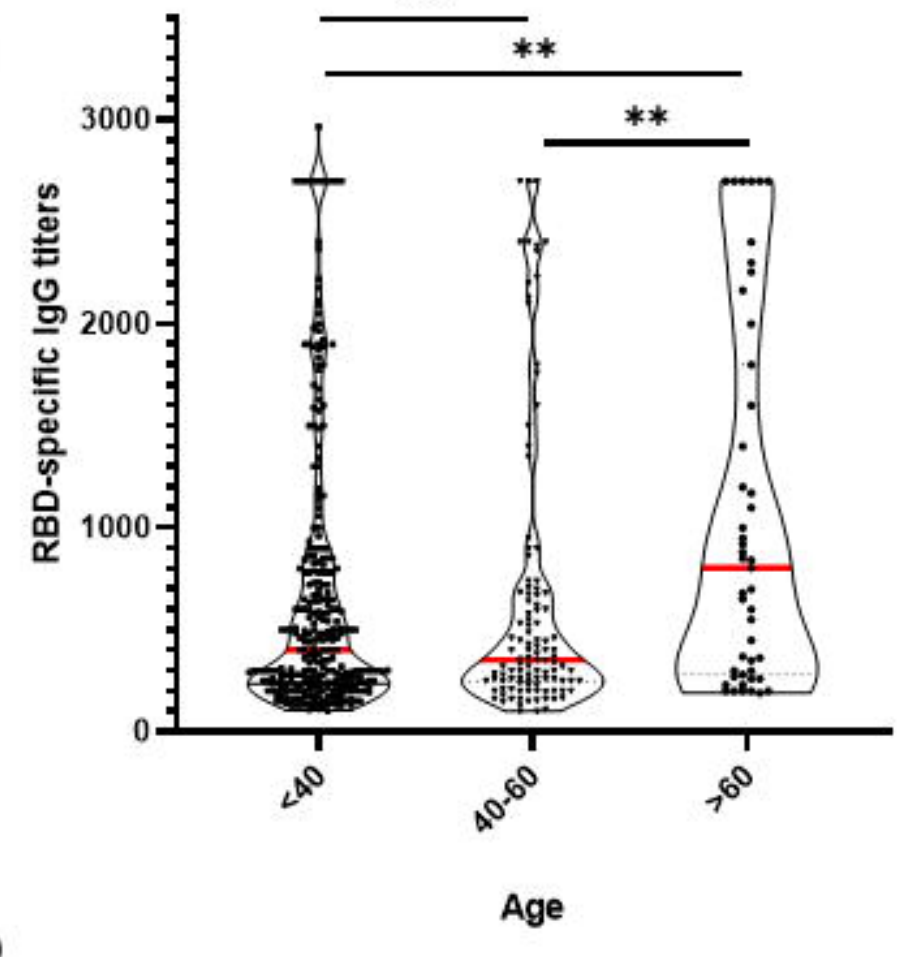

D

\begin{tabular}{lcccc}
\hline & \multicolumn{2}{c}{ Male } & \multicolumn{2}{c}{ Female } \\
\cline { 2 - 5 } & $\mathrm{n}$ & $\%$ & $\mathrm{n}$ & $\%$ \\
\cline { 2 - 5 } Positive & 278 & 51,1 & 177 & 90,3 \\
Negative & 266 & 48,9 & 19 & 9,7 \\
Total & 544 & 100 & 196 & 100 \\
\hline
\end{tabular}


Fig. 5

A

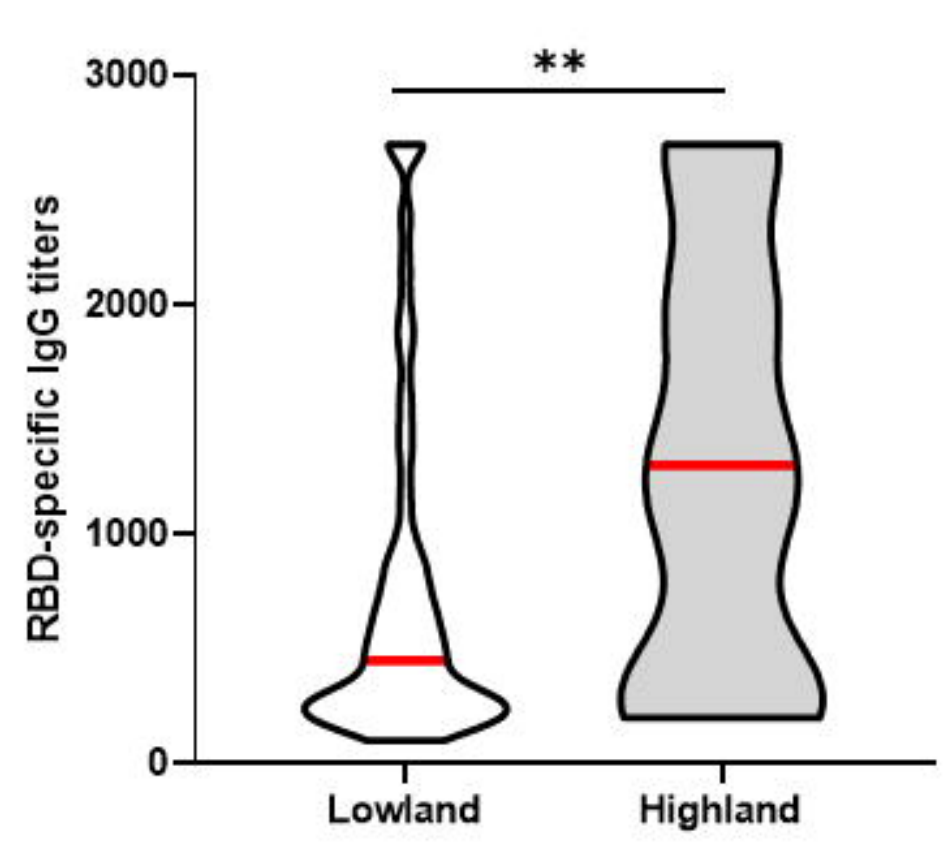

B

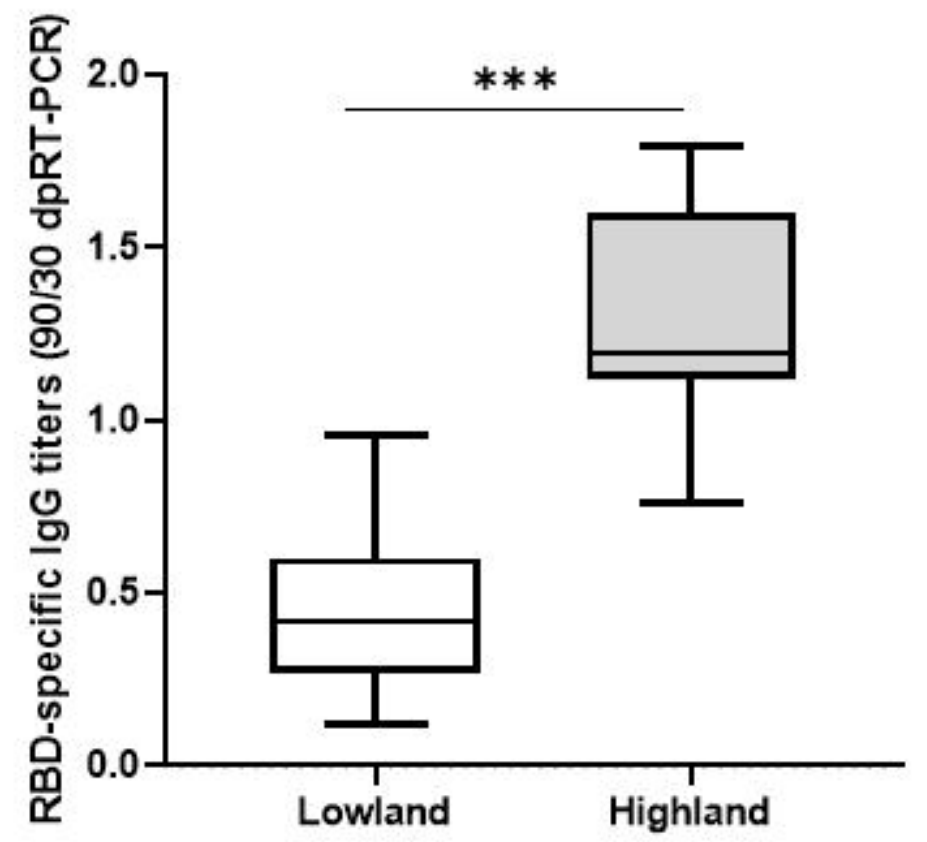

